\title{
Certified Vascular Nurse
}

National Cancer Institute

\section{Source}

National Cancer Institute. Certified Vascular Nurse. NCI Thesaurus. Code C71333.

A reg istered nurse with a vascular specialty encompassing caring for patients with a variety of arterial, venous and lymphatic diseases. 